\title{
A perfect refractory electrical storm by acute toxicity of accidental aconitine intake
}

\author{
Francesca Menichetti, ${ }^{1}$ Paola Bartalucci, ${ }^{2}$ Maria Luisa Matteucci, ${ }^{2}$ Luigi Gori, ${ }^{2}$ \\ Alessandra Ieri, ${ }^{3}$ Francesco Gambassi, ${ }^{3}$ Roberto Baronti, ${ }^{3,4}$ Simone Vanni, ${ }^{2}$ Guido Mannaioni, ${ }^{3}$ \\ Attilio Del Rosso ${ }^{1}$ \\ ${ }^{1}$ Cardiology Unit, San Giuseppe Hospital; ${ }^{2}$ Emergency Medicine Unit, San Giuseppe Hospital, Azienda USL \\ Toscana Centro, Empoli; ${ }^{3}$ Toxicology Unit, Azienda Ospedaliera Universitaria Careggi, Florence; \\ ${ }^{4}$ Toxicology Department, University of Florence; ${ }^{5}$ SOS Clinical Toxicology and Antidoping, Azienda USL \\ Toscana Centro, Florence, Italy
}

\begin{abstract}
A 51-years-old male presented with drooling, hematemesis and diarrhea in emergency department after accidental ingestion of homemade aconitine tincture. Examination revealed shock and initial ECG showed atrial fibrillation. The patient suddenly developed a pulseless wide QRS complex tachycardia requiring resuscitation maneuvers. The rhythm varied from monomorphic ventricular tachycardia to torsade de point to ventricular fibrillation. Repeated direct-current cardioversions were unsuccessful. After intravenous electrolyte correction and anti-arrhythmic drugs administration, a last direct-current shock was finally successful. Bradycardia and ventricular excitability persisted over 12 hours with gradual nor-
\end{abstract}

Correspondence:Francesca Menichetti, Cardiology Unit, San Giuseppe Hospital, Viale Boccaccio, 50053 Empoli, Italy

Tel.: +390571706834

E-mail: francesca.menichetti@uslcentro.toscana.it

Key words: Aconitum; toxicity; traditional medicine; cardiac arrhythmias; ventricular fibrillation.

Conflict of interests: No one. This work was not supported by any grant.

Availability of data and materials: All data underlying the findings are fully available.

Ethics approval and consent to participate: No ethical committee approval was required for this case report by the Department, because this article does not contain any studies with human participants or animals. Informed consent was obtained from the patient included in this study.

Consent for publication: The patient gave his written consent to use his personal data for the publication of this case report and any accompanying images.

Received for publication: 9 March 2021.

Revision received: 20 April 2021.

Accepted for publication: 30 April 2021.

This work is licensed under a Creative Commons Attribution 4.0 License (by-nc 4.0).

( Copyright: the Author(s), 2021

Licensee PAGEPress, Italy

Emergency Care Journal 2021; 17:9736

doi:10.4081/ecj.2021.9736 malization. Patient was discharged in normal sinus rhythm without any medication and on follow-up he was totally asymptomatic.

With the increasing popularity of traditional medicine, clinicians should be alerted to the risk of herbal substances with low margins of safety like Aconitum in order to allow correct diagnosis and treatment. The general public should be educated that herbal medicine is not always safe.

\section{Case Report}

A 51-years-old male with history of arterial hypertension presented in emergency department for epigastric burning, nausea and vomiting, of 2 hours duration. In triage ward patient had drooling, hematemesis and diarrhea and his vital signs were: blood pressure 118/80 $\mathrm{mmHg}$, pulse rate $69 \mathrm{bpm}$, and oxygen saturation $98 \%$ on room air. Patient doesn't speak the hospital native language but emergency physician asked to the patient's daughter if he has taken some toxic and she showed on her mobile a picture of a plant with purple flowers. The patient at night misunderstood hand-made content of a bottle and he accidentally drunk $100 \mathrm{~mL}$ of an aconitine decoction done for epidermal use. Through a research on the web the name of this plant was found to be Aconitum Napellus, a highly toxic flowering plant in the genus Aconitum of the family Ranunculaceae, also called monk's-hood or wolfsbane. Aconitum Napellus is also a very popular and dangerous medication in traditional Chinese medicine. After the toxic intake discovery, the physician consulted a tertiary medical center with expertise on poisoning management. They stressed to pay attention on cardiac and neurological aconitum toxic effect. Decontamination with nasogastric tube followed by activated charcoal was not recommended due to late time of presentation at the emergency department, 1 intense vomiting and hematemesis. Nonetheless, nasogastric tube was inserted in order to relieve nausea and vomiting. In Emergency Department ward the patient was anxious and sweating profusely, blood pressure dropped to $77 / 40 \mathrm{mmHg}$, pulse rate rose to $180 \mathrm{bpm}$ and bilateral miosis was present, initial ECG showed atrial fibrillation at $180 \mathrm{bpm}$ with wide QRS complex and repolarization abnormalities (Figure 1). Suddenly patients developed a pulseless wide QRS complex tachycardia requiring resuscitation maneuvers. The rhythm varied from monomorphic ventricular tachycardia to torsade de point to ventricular fibrillation. Repeated direct-current cardioversions (8 electrical interventions, Figure 2) were unsuccessful and the ventricular arrhythmia was incessant. Blood examination showed hypokalemia $(2.6 \mathrm{mEq} / \mathrm{L})$ and hypocalcemia $(\mathrm{Ca} 2+4,33 \mathrm{mg} / \mathrm{dL})$, so electrolyte correction was made. During CPR also sodium bicarbonate and high dose of intravenous magnesium sulphate has been administered. As anti- 
arrhythmic drugs, intravenous amiodarone and xylocaine had been given. All these infusions did not be able to fix the electrical instability until a Calcium intravenous rapid infusion has been given. A last direct-current shock was finally successful. The ECG after pulse recovery was junctional rhythm with bigeminal ventricular ectopy (Figure 3). In the next while other 3 single episodes of monomorphic ventricular tachycardia required direct-current cardioversions. Bradycardia and ventricular excitability persisted over $12 \mathrm{~h}$ with gradual normalization.

Toxicological test revealed high levels of blood aconitine (7 $\mathrm{ng} / \mathrm{mL}$ - normal values $<5 \mathrm{ng} / \mathrm{mL})$ and urinary mesacontine $(2,7$ $\mathrm{mg} / \mathrm{L}-$ normal values $<0,005 \mathrm{mg} / \mathrm{L}$ ) respectively. After 48 hour the values were normalized.

In the early phase after CPR the echocardiogram showed mild left ventricular hypertrophy (interventricular and posterior wall thickness $12 \mathrm{~mm}$ ) and mild reduction of ventricular systolic function (EF 48\%) without other significant abnormalities.

Patient was discharged in normal sinus rhythm on 5th day of hospitalization, without any medication. ECG at discharge showed normal sinus rhythm, with normal PR and QTc intervals and QRS duration. A complete recovery of left ventricular function (FE $60 \%$ ) was observed at echocardiogram. On follow-up after 1 month patient was totally asymptomatic.

\section{Discussion}

Aconitum is historically recorded as one of "The Four Pillars" of Traditional Chinese Medicine: ${ }^{2}$ it was mainly applicated for his antinociceptive and anti-inflammatory effects or local anesthetic properties. It is also used as traditional medicine in India and Japan. Aconitum toxicities, effectiveness, and phytochemistry are diversified on the base of its phylogeny. ${ }^{2}$ About 54 species of Aconitum have been chemically investigated and 3 major groups of alkaloids have been distinguished from 224 alkaloids isolated: monoterpene diester alkaloids, diterpene diester alkaloids and lipoalkaloids. ${ }^{3}$ They share a common C19-norditerpenoid skeleton, in which the $\mathrm{C} 8$ position is occupied by a hydroxyl, an acetoxy or a fatty acid acyl group, respectively. ${ }^{3}$ The toxicity of Aconitum mainly derives from the diester diterpene alkaloids, including aconitine, mesaconitine and hypaconitine. They can be decomposed into less or non-toxic derivatives through Chinese traditional processing methods (Paozhi), which play an essential role in detoxification. ${ }^{4}$

Hand-made aconitum tincture contains very high concentrations of aconite alkaloid, which are highly soluble in alcohol and lipids and rapidly absorbed from gastrointestinal tract. Aconitine's half-life is about 3 hours and alkaloid can be detected in blood and urine by liquid chromatography-mass spectrometry. The 19-carbon diterpenoid ring structure alkaloids are the most pharmacologically active and are capable of binding to excitable tissue. ${ }^{5}$ Onset is rapid, usually within a few minutes. Violent toxicity of aconites is well known from ancient times ("vegetal arsenic"), and it was used throughout the world and for centuries as a poison, to commit murder and suicide. Aconite roots are used only after soaking and boiling processing or decoction preparation, to reduce the toxic alkaloid content for less toxic and non-toxic derivates. The use of a dose larger than recommended, incorrect preparation and a narrow therapeutic index have been implicated in the toxic effects.

The lethal dose for adults is about $5 \mathrm{mg}$, but a dose of $1 \mathrm{mg}$ is often sufficient to cause severe cardiac rhythm disturbances and death. ${ }^{6-8}$ Severe poisoning has been caused even by ingestion of 0.2 mg pure aconitine, or by decoctions with $6 \mathrm{~g}$ of Aconitum roots. ${ }^{9}$
In 2 reported cases of voluntary intoxication and death, the autopsy revealed in both the same findings: blood congestion, hemorrhages in the lung tissue, lung edema and brain edema. ${ }^{10}$ Toxicity commonly begins within 30 to 90 minutes after ingestion and may last for $24-48$ hours. ${ }^{11}$ The overall in-hospital mortality is $5.5 \%$.



Figure 1. ECG at hospital admission, with fast heart rate atrial fibrillation. Please note the half voltage in the precordial leads due to a recording error in an emergency situation. The widening of the final component of QRS complex and the spreading of repolarization abnormalities should make you suspect of cardiac cellular action potential modification.
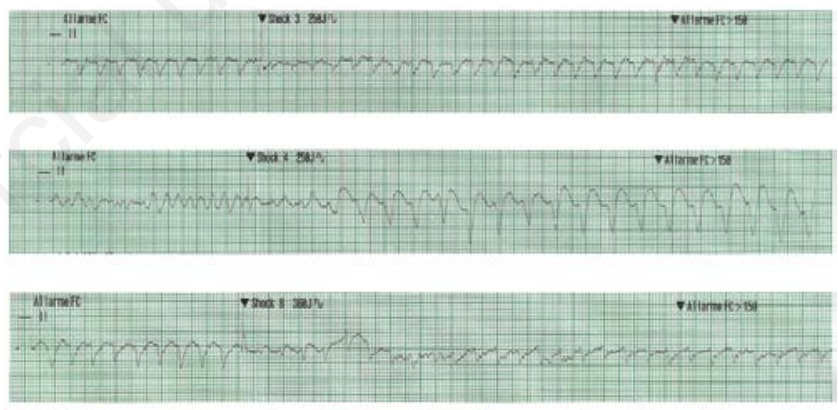

Figure 2. Some of the repeated emergence direct-current cardioversions, unsuccessful to convert the incessant ventricular arrhythmia.

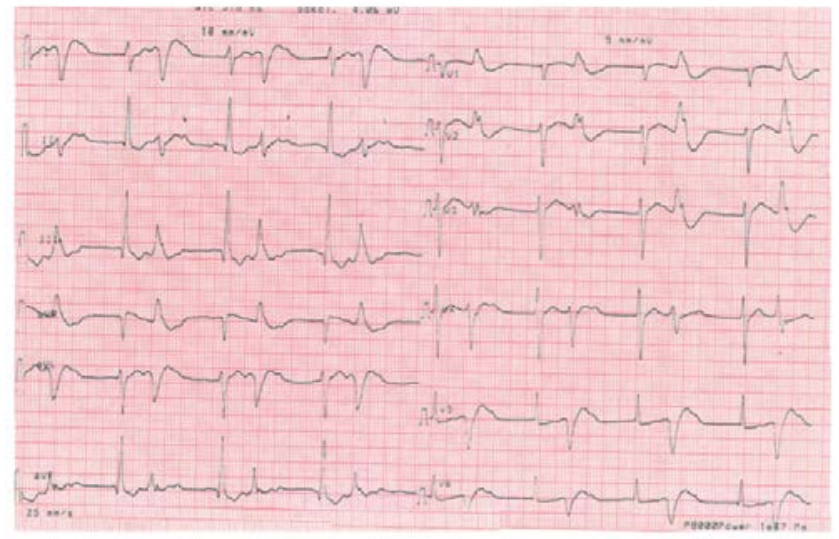

Figure 3. After arrhythmic storm termination the rhythm was junctional with bigeminal ventricular ectopy, as result of the prevalence of aconitine bradycardic action. Please note the half voltage in the precordial leads due to a recording error in an emergency situation. 
The main causes of death are refractory ventricular arrhythmias, asystole, refractory shock or respiratory paralysis occurring within the first 24 hours. Aconitine's toxicity is characterized by a burning sensation of the lips, tongue, mouth, and throat almost immediately following ingestion; it may be followed by numbness of the oral cavity of the oral cavity and throat and difficulty to speech; dizziness, syncope, palpitation may occur. ${ }^{12}$ Aconitine in very high concentration blocks the release of acetylcholine reducing the vagotonic effect on myocardium (anticholinergic effects), while at lower concentration increases acetylcholine release. Hypotensive and bradycardic action are due to activation of the ventromedial nucleus of the hypothalamus, ${ }^{13}$ as well as to the increase of vagal tone. Aconitine and other alkaloids can induce strong contractions of the ileum through acetylcholine release from the postganglionic cholinergic nerves causing gastrointestinal symptoms such as nausea, vomiting, and diarrhea. ${ }^{14}$

The aconitine toxin exhibits a high binding affinity for voltagedependent sodium channels. The binding of these alkaloids promotes persistent channel opening, with a prolonged increase in intracellular $\mathrm{Na}^{+}$and $\mathrm{Ca}^{2+}$, and subsequent sustained membrane depolarization. ${ }^{15}$ Aconitine has been shown to hold in the open state also the potassium channel responsible for the rapid delayed rectifier current (IKr) and the ultra-rapid delayed rectifier current (IKur). ${ }^{16}$ This effects might lead to further prolongation of the action potential, and thus further increase the arrhythmogenesis. The net effect of the above mechanisms leads to arrhythmogenesis via early and delayed after-depolarization. Aconitine produces persistent flutter and fibrillation of cardiac and skeletal muscles resulting in a life threatening cardiac arrhythmias. Most fatal cases have been determined by ventricular tachycardia or respiratory paralysis.

In 65 case reports of probable aconite poisoning that resulted in ventricular dysrhythmias 54 developed ventricular tachycardia, six developed torsades de pointes, 15 patients developed ventricular fibrillation, 10 developed ventricular ectopies and one developed a broad complex tachycardia not otherwise specified. Ten patients died, resulting in a $15 \%$ mortality. ${ }^{17}$ Flecainide was administered on seven occasions and was associated with a return to sinus rhythm in six of these patients. Amiodarone was given on 20 documented occasions, with reversion to sinus rhythm occurring on 11 of these administrations. Lidocaine was employed on 30 occasions with documentation of conversion to sinus rhythm on only one of these. ${ }^{11}$ The ventricular tachyarrhythmias responded poorly to direct-current cardioversion: DC shock was applied 30 times but the restoration of sinus rhythm has been observed only on five occasions. Prolonged CPR was reported as having been used in 15 patients, with a conversion to sinus rhythm in nine patients. ${ }^{11}$ Prolonged CPR should be considered central in the treatment of ventricular arrhythmias precipitated by aconite as it may allow time for the alkaloids to be naturally removed from the plasma. As a last effort, mechanical support with the use of cardiopulmonary extracorporeal bypass might be considered for circulatory support, as the inactivation of voltage-dependent $\mathrm{Na}^{+}$ channels from an aconitine ingestion is generally transient.

In our experience high dose of Calcium administration has been followed by the reversal of aconitum arrhythmic refractoriness and a final direct-current shock successful. This may suggest that, even if aconitine toxin exhibits a high binding affinity for voltage-dependent sodium channels, Sodium acts only consistently with Calcium.

As differential diagnosis, is important to suspect aconite poisoning when muscarinic intoxication clinical signs are present, especially in Asian people. When a patient presents with numb- ness, muscle weakness, unexplained arrhythmia or ventricular tachycardia refractory to direct-current cardioversion and standard anti-arrhythmic drugs, aconite poisoning should be considered and routine blood and urine chemical screening tests should be able to detect such lethal plants. ${ }^{18}$

\section{Conclusions}

With the increasing popularity of traditional medicine, the professional community and the public should be alerted to the risk of herbal substances with low margins of safety like Aconitum. Although reports of aconite poisonings are actually rare in western countries, the incidence could change with the increasing popularity of herbal medicines and migratory flows of Chinese population. ${ }^{19,20}$ The general public should be educated that Chinese medicine is not always benign and safe, and consulting a medicine practitioner before taking herbs is always advisable. Because of the potential toxicity, quality control of marketed herbal drugs and their products is essential to ensure that the alkaloid levels are within safe limits. Awareness and knowledge of common herbal poisoning among clinicians can allow correct diagnosis and a timely treatment of the poisoned patients.

\section{References}

1. Benson BE, Hoppu K, Troutman WG, et al. Position paper update: gastric lavage for gastrointestinal decontamination. Clin Toxicol (Phila) 2013;51:140-6.

2. Xiao P, Wang F, Gao F, et al. A pharmacophylogenteic study of Aconitum L (Ranunculaceae) from China. Acta Phytotaxonomica Sinica 2006;44:1-46.

3. Singhuber J, Zhu M, Prinz S, et al. Aconitum in Traditional Chinese Medicine-A valuable drug or anunpredictable risk? J Ethnopharmacology 2009;126:18-30.

4. Singhuber J, Zhu M, Prinz S, Kopp B. Aconitum in Traditional Chinese Medicine-A valuable drug or an unpredictable risk? J Ethnopharmacol 2009;126:18-30.

5. Liu S, Li F, Li Y, et al. A review of traditional and current methods used to potentially reduce toxicity of Aconitum roots in Traditional Chinese Medicine. J Ethnopharmacol 2017;207:237-50.

6. Fujita Y, Terui K, Fujita M, et al. Five cases of aconite poisoning: toxicokinetics of aconitines. J Anal Toxicol 2007;31:1327.

7. Bicker W, Monticelli F, Bauer A, et al. Quantification of aconitine in post-mortem specimens by validated liquid chromatography-tandem mass spectrometry method: three case reports on fatal 'monkshood' poisoning. Drug Test Anal 2013;5:753-62.

8. Van Landeghem AA, De Letter EA, Lambert WE, et al. Aconitine involvement in an unusual homicide case. Int $\mathrm{J}$ Legal Med 2007;121:214-9.

9. Tai TY, But PP, Young K, Lau CP. Cardiotoxicity after accidental herb-induced aconite poisoning. Lancet 1992;340:1254-6.

10. Veit F, Gürler M, Nebel A, et al. Intentional ingestion of aconite: Two cases of suicide. Forensic Sci Int Rep 2020;2:100158.

11. Fujita Y, Terui K, Fujita M, et al. Five cases of aconite poisoning: toxicokinetics of aconitines. J Anal Toxicol 2007;31:132 7.

12. Tai YT, But PPH, Young K, Lau CP. Cardiotoxicity after acci- 
dental herb-induced aconite poisoning. Lancet 1992;340: 1254-56.

13. Yamanaka H, Doi A, Ishibashi $H$, et al. Aconitine facilitates spontaneous transmitter release at rat ventromedial hypothalamic neurons. Br J Pharmacol 2002;135:816-22.

14. Chan TY. Aconite poisoning. Clin Toxicol (Phila) 2009;47:279-85.

15. Friese J, Gleitz J, Gutser UT, et al. Aconitum sp. alkaloids: the modulation of voltage-dependent $\mathrm{Na}+$ channels, toxicity and antinociceptive properties. Eur J Pharmacol 1997;337:165-74.

16. Wang YJ, Chen BS, Lin MW, et al. Time-dependent block of ultrarapid-delayed rectifier $\mathrm{K}+$ currents by aconitine, a potent cardiotoxin, in heart-derived $\mathrm{H} 9 \mathrm{c} 2$ myoblasts and in neonatal rat ventricular myocytes. Toxicol Sci 2008;106:454-63.

17. Coulson JM, Caparrotta TM, Thompson JP. The management of ventricular dysrhythmia in aconite poisoning. Clin Toxicol (Phila) 2017;55:313-21.

18. Li S, Chen D. Rapid determination of Aconitum alkaloids from human urine by UHPLC-HRMS. J Anal Toxicol 2017;41:6117.

19. Dwivedi S, Aggarwal A. Cardiotoxicity From "Safe" Herbomineral Formulation. Trop Doct 2011;41:113-5.

20. Moritz F, Comagnon P. Severe acute poisoning with homemade Aconitum Napellus capsules. Toxicokinetic Clin Date 2009;43:873-6. 